\title{
Compensability of Errors in Manufacturing of Pushing Chain
}

\author{
Leiyu Zhang \\ Department of Mechanical Design and Automation, \\ BeiHang University, Beijing, China \\ e-mail: zhangleiyu1988@126.com
}

\author{
Yang Yang \\ Department of Mechanical Design and Automation, \\ BeiHang University, Beijing, China \\ e-mail: yang_mech@buaa.edu.cn
}

\begin{abstract}
The pushing chain is a novel telescopic mechanism used to push the object from one position to another rapidly. The position precision of the pushing chain influenced by various factors is very important to accomplish the task of pushing the object. To compensate the clearances and machining errors, the mathematical model of position precision under the static condition is established. The influence of each factor is obtained through the numerical simulation. Furthermore, the bended deformation of the extended chain is calculated using the mechanics of materials method. According to the influences and the bended deformation, an error compensation method for a certain pushing distance is proposed so as to improve the position precision of chain head. In addition, the chain with compensation is possible to be extended in a straight line. This research provides theoretical and methodological guidance for compensability of errors in manufacturing of pushing chain.
\end{abstract}

Keywords- pushing chain, telescopic mechanism, position precision, error compensation method

\section{INTRODUCTION}

The pushing chain is an important element of telescopic mechanism used to push the object from one position to another rapidly. Unlike the conventional roller chain, the pushing chain, which is an open structure, has the bended directionality. The chain can only be bended in the opposite direction of gravity in the horizontal direction. Then it can be rolled up inside a case with arbitrary shape in a compact manner. Therefore, the pushing chain mechanism with compact volume and extreme telescopic distance is suitable for the longdistance pushing in a constrained space. In addition, the pushing chain possesses high speed performance.

As an important element of the telescopic mechanism, the pushing chain usually connects the base portion and the end-effector. There are several types of this mechanisms used to move remote handling equipment. Lee and Park [1] disclosed a telescopic tube set for a bridge transport system, which includes several cylindrical tubes and extension/retraction lines. D. Lee and Chang [2] designed a wire-driven bidirectional telescopic mechanism consisting of stages and steel wires actuated by one servo motor. A telescopic mechanism developed by Enders et al. extends by rapidly introducing a fluid and retracts by venting the fluid [3]. The scissors aerial work platform is a typical type of telescopic equipment that is widely used for high altitude operation and maintenance [4]. Another linear-motion telescopic mechanism invented by Kawabuchi [5] includes a plurality of block members. An arbitrary length is achieved in such a manner that block members are rigidly connected with each other forming a telescopic arm However, in these types of telescopic mechanisms above, the volume of the base portion is quite large in order to achieve sufficient stiffness.

The objective of this paper is to analyze the position precision of the pushing chain at a certain pushing distance. The mathematical model of position precision influenced by the hinge clearances and machining errors is established. The bended deformation of the chain is calculated using the mechanics of materials method. According to the mathematical model and bended deformation, an error compensation method is proposed to improve the position precision of the pushing chain and keep the chain extended in a straight line.

\section{STRUCTTURE OF PUSHING CHAIN}

The pushing chain contains double-row roller, bushing, link, pin and ring, as shown in Fig .1(a). There are actually two types of links alternating in the pushing chain. Both the narrow and wide links pivot on the same bushing. Two rollers engaged with the sprocket are mounted at each end of this bushing. The pin inserted in the bushing rivets the bushing and ring together. Then the double-row roller is assembled. Application of double-row roller improves the anti-wear performance of the proposed mechanism.

Two contact surfaces (Fig .1(a)), one on a wide link and the other on the adjacent narrow link, prevent the adjacent links rotating clockwise. The adjacent links only rotate around the pivot $O$ anticlockwise, which is called the bended directionality of the pushing chain. Consequently, the pushing chain can be stretched out in a straight line under the thrust force imposed by drive sprocket and its own gravity when it pushes the object in horizontal direction.

The pushing chain mechanism consists of drive sprocket, pushing chain, guide groove and helicoids case, as shown in Fig .1(b). The track of helicoids case for storing the pushing chain is Archimedes spiral. The drive sprocket is made up of two identical sprockets which are welded together. The sprocket engages with double-row rollers of chain links. The pushing chain is extended out along the guide groove and then retreated into the 
helicoids case. The extended part is supported by the chain link staying in the groove. The chain head which is installed at the front end of the extended chain has contact with the object during the pushing process.

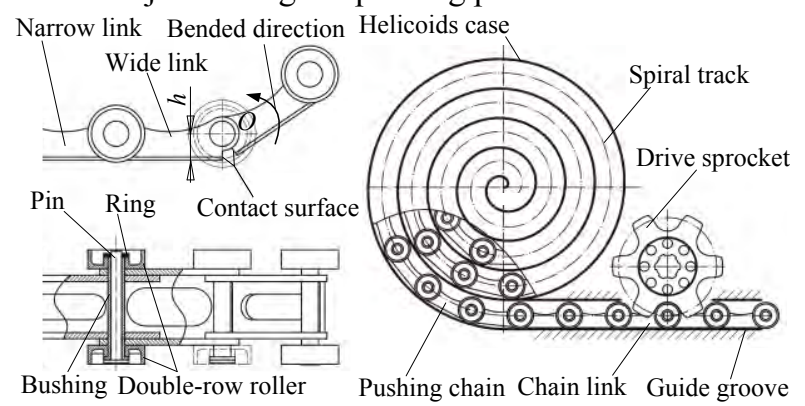

(a)

(b)

Figure 1. Structure of pushing chain mechanism

Because of the hinge clearances of machining errors, the chain head of the extended chain droops. Even the extended chain can not push the object to the object location precisely. Hence, the position precision of the chain head is important to the pushing chain. The influences of the errors should be analyzed for the compensability of errors in the manufacturing of pushing chain.

\section{MATHEMATICAL MODEL OF POSITION PRECISION OF PUSHING CHAIN}

There are two main factors influencing the position precision of the pushing chain. The first one is the clearances existing in hinges consisting of a link and a bushing. The second one is the machining errors of the bottoms of links. The mathematical models of position precision under the static condition will be introduced in this section.

In order to analyze conveniently, the chain strand is approximated as a series of point mass lumped at the roller centers $[9,10]$. The total mass of the link, roller, bushing, pin and ring is denoted as $m$, which is called the point mass.

\section{A. Model of Clearance $e_{d i}$ on Position Precision}

There are a wide link and a narrow one pivoting around the same bushing. For simplifying formula deduction procedures, assume that both the wide link and narrow link have equal clearance $e_{d i}$ with regard to the bushing, as shown in Fig .2(a). Under such assumption, the clearance $e_{d i}$ is given by

$$
e_{d i}=r_{w(i+1)}-r_{b i}=r_{n i}-r_{b i}
$$

where $r_{w(i+1)}$ and $r_{n i}$ are the bore radii on the wide link $i+1$ and narrow link $i$ respectively; $r_{b i}$ is the outer radii of bushing $i, i$ is the serial number of links numbered sequentially from 1 to 41 .

Due to the existence of clearance, the contact between the link and bushing is linear contact instead of surface contact. The direction of contact force between the link $i$ and bushing $i$ is identical to that of the resultant force $\boldsymbol{F}_{i}$, acting upon the link $i$. Meanwhile, the pivot $O_{i}$ falls on the direction of force $\boldsymbol{F}_{i}$. The contact angle $\theta_{i}$ of force $\boldsymbol{F}_{i}$ is the angle relative to the horizontal direction, as shown in
Fig .2(b). The link $i+1$ and link $i$ are separated along the direction of force $\boldsymbol{F}_{i}$ and the point $O_{i}^{\prime}$ is the new pivot of the link $i$. The separation distance $e_{s i}$ is given by

$$
e_{s i}=2 e_{d i}
$$

$e_{h i}$ and $e_{v i}$ are components in the horizontal and vertical direction respectively, where

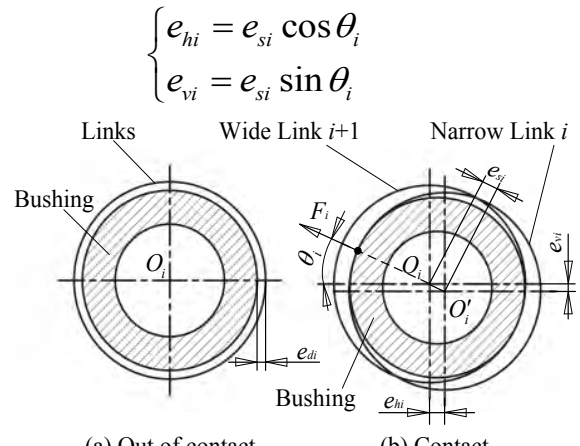

(a) Out of contact

(b) Contact

Figure 2. Contact of hinges with clearances

Under their own gravity, the bottom of each extended link contacts and supports each other to keep the extended part in a straight line. The length $l$ of the bottom which is equal to the chain pitch $P$ generally has machining error $e_{l i}$. Thus the link $i$ rotates around the pivot $O_{i}$ by the inclination angle $\delta_{i}$ relative to the link $i+1$ when their bottoms contact closely, as shown in Fig .3. In other words, the posture of the link $i$ is determined by the angle $\delta_{i}$.

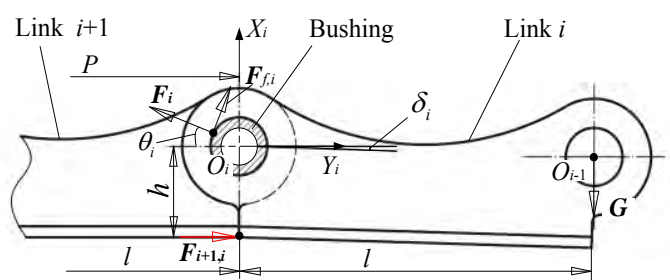

Figure 3. Contact among the bottoms of extended links

The local coordinate frame $X_{i} O_{i} Y_{i}$ is fixed on the hinge pivot of the link $i+1$ which stays in the horizontal position. $G$ is the gravity of each link and $M_{i}$ is the sum of moments which are induced by the gravities of the former $i$ links on the pivot $O_{i}$. The reacting force $\boldsymbol{F}_{i+1, i}$ is the force that the bottom of link $i+1$ acts on the link $i$ (Fig .3). $F_{f, i}$ is the friction between the bushing $i$ and the link $i$. The forces and moments on link $i$ are in equilibrium relative to the pivot $O_{i}$, respectively.

Equilibrium gives

$$
\left\{\begin{array}{l}
F_{i} \tan \theta_{i}=i G \\
F_{i+1, i} h+F_{f, i}+M_{i}=0
\end{array}\right.
$$

where

$$
F_{f, i}=2 \mu F_{i} r_{b i}
$$

$\mu$ is the friction coefficient in the contact area.

All the extended links are connected in sequence and the link $i$ rotates around the pivot $O_{i}$ by angel $\delta_{i}$ relative to the link $i$. Each extended link produces an eccentric moment on the pivot $O_{i}$ under its own gravity $G$. 
Furthermore, the influence of the inclination angle $\delta_{i}$ on the moment $M_{i}$ is taken into account. The complete induction is adopted in the deviation of the moment $M_{i}$.

For a general $i$-link chain span, the resultant moment $M_{i}$ is obtained and then given below.

$$
\begin{aligned}
M_{i}= & G \frac{P}{2} \cos \delta_{i} \\
+ & G\left[P \cos \delta_{i}+\frac{P}{2} \cos \left(\delta_{i}+\delta_{i-1}\right)\right] \\
& \mathrm{M} \\
+ & G\left[P \cos \delta_{i}+P \cos \left(\delta_{i}+\delta_{i-1}\right)+\mathrm{L}\right. \\
& \left.+\frac{P}{2} \cos \left(\sum_{j=i}^{i} \delta_{j}\right)\right] \\
= & G \sum_{j=1}^{i}\left\{\frac{[2(i-j)+1]}{2} P \cos \left(\sum_{k=i-j+1}^{i} \delta_{k}\right)\right\}
\end{aligned}
$$

Substitute equation (5) into (4), it is obtained that

$$
\tan \theta_{i}=\frac{2 i h}{P} \mathrm{~g} \frac{1}{\sum_{j=1}^{i}\left\{[2(i-j)+1] \cos \left(\sum_{k=i-j+1}^{i} \delta_{k}\right)\right\}}
$$

\section{B. Model of Machining Error $e_{l i}$ on Position Precision}

The machining error $e_{l i}$ of dimension $l$ (Fig .3) is another main factor affecting position precision of the pushing chain. Since a number of links are machined simultaneously using wire electrical discharge machining, it can be assumed that the sum of the right unilateral error $e_{l(i+1)}^{\prime}$ of the link $i+1$ and left unilateral error $e_{l i}^{\prime}$ of the link $i$ is equal to the machining error $e_{l i}$, where

$$
e_{l i}=e_{l(i+1)}^{\prime}+e_{l i}^{\prime}
$$

The new pivot $O_{i}^{\prime}$ on the link $i$ is defined as coordinate origin, and then the local coordinate frame $X_{i}^{\prime} O_{i}^{\prime} Y_{i}^{\prime}$ is constructed, as shown in Fig .4. Due to the existence of clearances $e_{d i}$ and machining errors $e_{l i}$, the link $i$ (blue profile) can rotate around the pivot $O_{i}^{\prime}$ clockwise by angle $\delta_{i}$. The inclination angle $\delta_{i}$ of the link $i$ is composed of $\delta_{i}^{\prime}$ and $\delta_{i}^{\prime \prime}$, which are given below

$$
\left\{\begin{array}{l}
\tan \delta_{i}^{\prime}=\frac{e_{l(i+1)}^{\prime}-e_{h i}}{h-e_{v i}} \\
\tan \delta_{i}^{\prime \prime}=\frac{e_{l i}^{\prime}}{h}
\end{array}\right.
$$

Using the addition theorem of trigonometric function, the equations (8) are simplified as

$$
\begin{aligned}
\tan \delta_{i} & =\tan \left(\delta_{i}^{\prime}+\delta_{i}^{\prime \prime}\right)=\left(\tan \delta^{\prime}+\tan \delta^{\prime \prime}\right)\left(1-\tan \delta^{\prime} \tan \delta_{i}^{\prime \prime}\right) \\
& \left.=\frac{1}{h}\left(\frac{e_{l(i+1)}^{\prime}-e_{h i}}{1-\frac{e_{v i}}{h}}\right) \notin 1-\frac{e_{l i}^{\prime}\left(e_{l(i+1)}^{\prime}-e_{h i}\right)}{h^{2}\left(1-\frac{e_{r i}}{h}\right)}\right]
\end{aligned}
$$

s.t. $\frac{e_{v i}}{h}<<1, \frac{e_{r i}}{h}<<1$ and $e_{l i}<<h$, the equation (9) can be further simplified as

$$
\tan \delta_{i}=\frac{e_{l(i+1)}^{\prime}-e_{h i}+e_{l i}^{\prime}}{h}=\frac{e_{l i}-e_{h i}}{h}
$$

Substituting equations (2) and (3) into (6) and (10), the angles $\theta_{i}$ and $\delta_{i}$ are obtained in sequence.

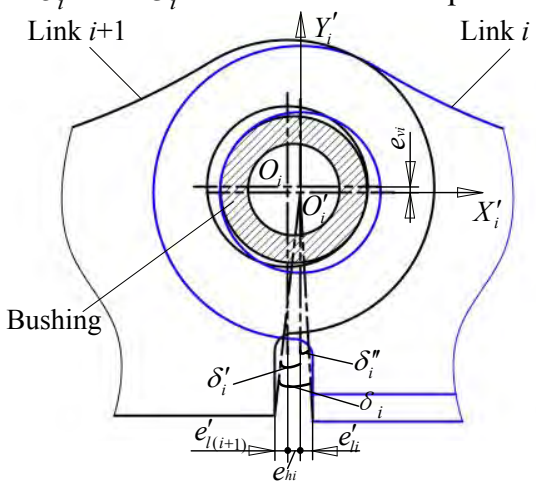

Figure 4. Contact relationship between bottoms of two links

\section{Deviation $\gamma_{i}$ of Each Link}

Due to the influences of clearances $e_{d i}$ and machining errors $e_{l i}$, the pushing chain may not be stretched out horizontally in a straight line. The deviation $\gamma_{i}$ of the link $i$ from the pushing direction is normally nonlinearly distributed. The clearance $e_{d i}$ and the inclination angle $\delta_{i}$ of the link $i$ are the main factors affecting the deviation $\gamma_{i}$. The deviation $\gamma_{i}$ is deduced by the iterative method, where

$$
\gamma_{i}=\sum_{j=i}^{40}\left(e_{d j} \operatorname{gsin} \theta_{j}\right)+P \mathrm{~g} \sum_{k=1}^{41-i} \sin \left(\sum_{m=41-k}^{40} \delta_{m}\right)
$$

\section{BENDED DEFORMATION OF EXTENDED CHAIN}

In practical assembly, one end of the pushing chain is fixed and the other end is free. The descend height of free end is measured to examine whether the chain is qualified or not. Therefore, the extended part may be simplified as the cantilever beam model in no-load operation. The link staying in the guide groove is approximated as the fixed end. Correspondingly, the chain head can be regarded as the free end. $L$ is the length of extended chain. The gravity of extended chain is simplified as the uniform loading $\boldsymbol{q}$ acting on the beam cantilever. In addition, the gravity of chain head $\boldsymbol{G}_{h}$ acts on the free end as a concentrated load, as shown in Fig .5.

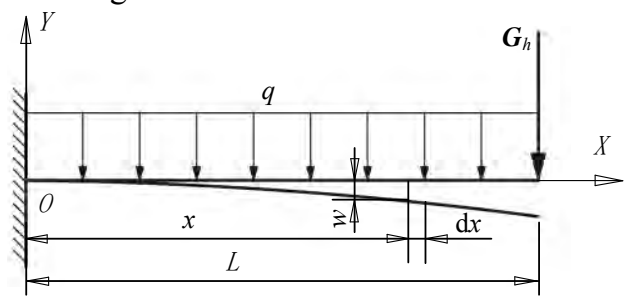

Figure 5. Cantilever beam model of the extended chain

Using the mechanics of material method, the deflection curve $w(x)$ for the beam cantilever is deduced

$$
w(x)=-\frac{G_{h} x^{2}}{6 E I}(3 L-x)-\frac{q x^{2}}{24 E I}\left(x^{2}-4 L x+6 L^{2}\right)
$$


where $x$ is the horizontal coordinate in the coordinate system $X O Y ; w$ and $E I$ are the deflection and bended rigidity of the beam cantilever, respectively.

Therefore, the bended deformation of the extended chain can be obtained by substituting given parameters into function (12).

Then, differentiate equation (12) with respect to $x$

$$
w^{\prime}(x)=-\frac{G_{h}}{2 E I}\left(2 L x-x^{2}\right)-\frac{q}{6 E I}\left(x^{3}-3 L x^{2}+3 L^{2} x\right)
$$

In the cantilever beam model, the extended chain consists of $n$ links and the inclination angle $\psi_{i}$ and $\psi_{i-1}$ of two adjacent links can be obtained through the following equation

$$
\left\{\begin{array}{l}
\tan \psi_{i}=w^{\prime}\left(x_{i}\right) \\
\tan \psi_{i-1}=w^{\prime}\left(x_{i-1}\right)
\end{array}\right.
$$

where $x_{i}=(n-i+1) P$ and $x_{i-1}=(n-i) P$. Thus, the relative inclination $\delta_{i}^{\prime}$ can be acquired using the knowledge of trigonometric function, where

$$
\delta_{i}^{\prime}=\psi_{i+1}-\psi_{i}
$$

Due to the fact that the extended chain is bended downwards under its own gravity, the relative inclination $\delta_{i}^{\prime}$ is always less than zero.

Substitute the main parameters into equations (14) and (15), and the inclination angle $\delta_{i}^{\prime}$, determined by the material characteristics of the pushing chain, may be obtained. If the chain is extended in a straight line, the bended deformation should be compensated through suitable clearances $e_{d i}$ and machining errors $e_{l i}$. Hence, when the clearances $e_{d i}$ remain constant, the machining error $e_{l i}$ of each link can be calculated by substituting the angles $\delta_{i}^{\prime}$ into equation (10).

\section{RESULTS AND ANALYSIS}

In this section, the position precision of the pushing chain for a certain pushing distance is calculated and analyzed. The calculation algorithm for the mathematical model above is programmed by use of MATLAB. For this helicoids pushing chain mechanism, the pushing distance is $2000 \mathrm{~mm}$ and there are 40 links in the extended part.

\section{A. Influence of the Clearance $e_{d i}$}

Since the links are in clearance fit with bushings, the clearance $e_{d i}$ exists definitely. The contact angle $\theta_{i}$ and the inclination angle $\delta_{i}$ are influenced by clearances $e_{d i}$ and machining errors $e_{l i}$. In order to more clearly understand the influence of $e_{d i}$, it is assumed that $e_{l i}=0$.

Fig .6 shows the contact angle $\theta_{i}$ at two different clearances $e_{d i}$. It is interesting to notice that there are three processes in the curves of the contact angle $\theta_{i}$ : an initial fast decay and an intermediate decay, followed by a long process of slow decay. The angle $\theta_{i}$ will eventually approach a constant value with the increase of the link number. It could also be seen that the two curves of $\theta_{i}$ overlap and the magnitude of $e_{d i}$ has little effect on the angle $\theta_{i}$.
Fig .7 shows the inclination angle $\delta_{i}$ of the link $i$ at four different clearances $e_{d i}$. For convenient observation, the semilog coordinate is adopted instead of Cartesian coordinate. It is obvious that the change trend of $\delta_{i}$ is similar to that of $\theta_{i}$. However, the magnitude of $\delta_{i}$ rises with the increment of the link number and the magnitude of $\delta_{i}$ is proportional to the clearance $e_{d i}$.

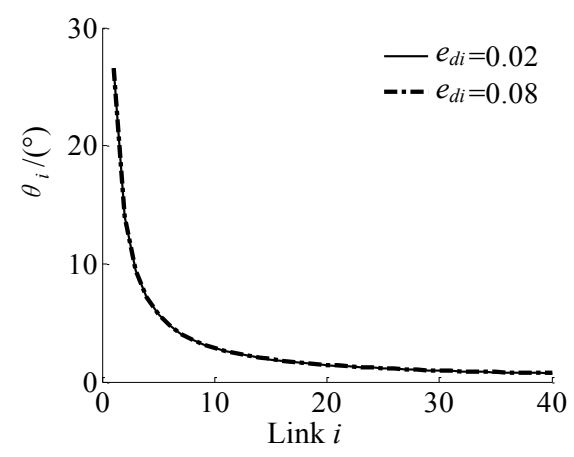

Figure 6. Contact angle $\theta \mathrm{i}$

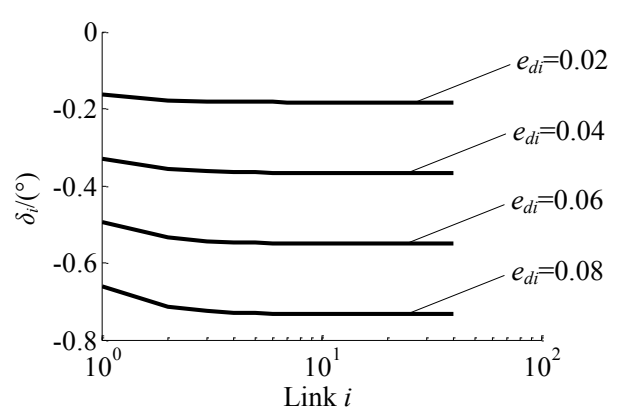

Figure 7. Inclination angle $\delta i$ at four different clearances edi

Fig .8 shows the deviations $\gamma_{i}$ of the link $i$ at different clearances $e_{d i}$. It could be seen that the deviation is in parabolic curve relationship with the link number. The value of deviation $\gamma_{i}$ increases with the decrease of the link number. The link 1 connecting the chain head has the maximum deviation, whereas the link 40 adjacent to the guide groove has the minimum deviation. Additionally, the values of whole deviation rise along with the increment of clearance $e_{d i}$.

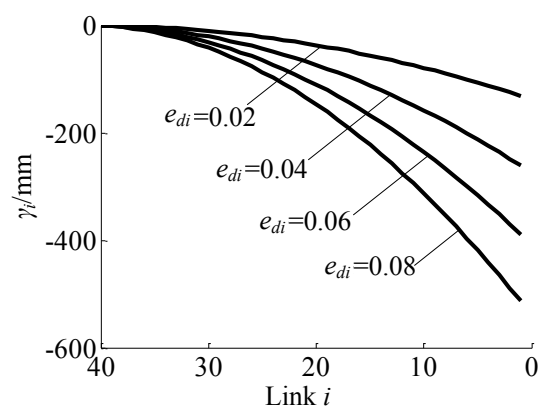

Figure 8. Deviation $\gamma$ i at four different clearances edi

\section{B. Influence of the Machining Errors $e_{l i}$}

Through the numerical simulation, it is found that the magnitude of machining error $e_{l i}$ has little effect on the contact angle $\theta_{i}$ as well. The change trend of $\theta_{i}$ influenced 
by the errors $e_{l i}$ is similar to that of angel $\theta_{i}$ in Fig .7. Hence the analysis of the angle $\theta_{i}$ is omitted here due to limited space.

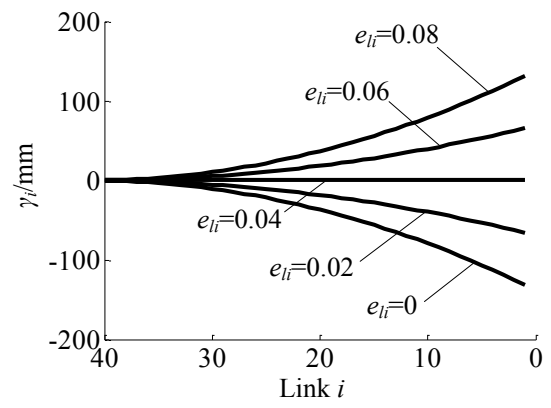

Figure 9. Deviation $\gamma \mathrm{i}$ at edi $=0.02$

Fig 9 shows the deviation $\gamma_{i}$ of the link $i$ at five different machining errors $e_{l i}$ at $e_{d i}=0.02$. It is interesting to notice that the deviation of the whole extended chain increases with the increase of machining errors $e_{l i}$. Besides, the shape of the chain changes from bended downwards to bended upwards gradually. It could be seen that the extended chain is approximated to a straight line at $e_{l i}=0.04$. Hence, when the machining error $e_{l i}$ is about twice the clearance $e_{d i}$, it has the best compensation effect without considering other influences. Besides, the influence of clearance $e_{d i}$ can be compensated by the machining errors $e_{l i}$

\section{Realization of high position precision of the chain}

If the chain is extended in a straight line at $e_{d i}=0.02$, the clearances $e_{d i}$ and the bended deformation should be compensated by the machining errors $e_{l i}$. The compensation values of machining errors $e_{l i}$ are calculated form equations (14), (15) and (10), as shown in Fig .10. Therefore, it is better to increase the machining errors $e_{l i}$ of the links close to the guide groove and decrease that of the links adjacent to the chain head.

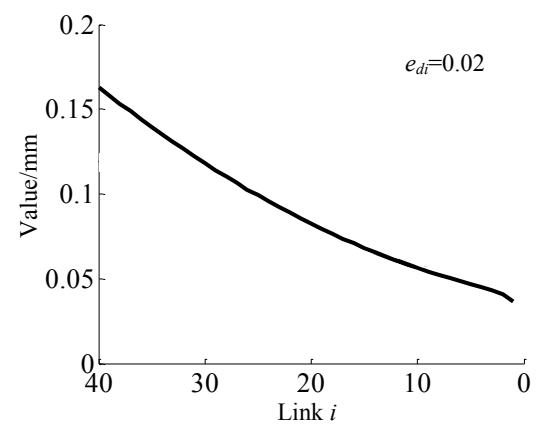

Figure 10. Machining errors eli

Curve 4 is the deviation curve of the extended chain at $e_{d i}=0.02$ and the compensation errors $e_{l i}$. Then, curve 5 is the superposition of curve 4 and curve $w(x)$, as shown in Fig .11. Besides, the coordinates of the links with the maximum deviation and the chain head are marked. The extended chain can approximately keep a straight line when it pushes the object to the target location. The extended chain with the error compensation possesses an excellent axial rigidity along the pushing direction. In addition, the position precision of the chain head is possible to be significantly improved.

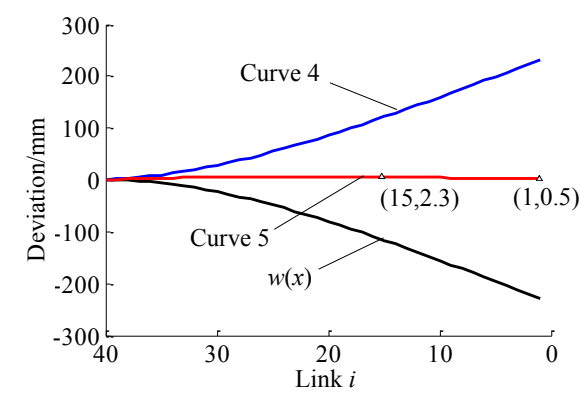

Figure. 11. Compensation of the clearances edi and the bended deformation

\section{CONCLUSION}

In this paper, the structure of the pushing chain is developed. Because of the bended directionality there are two main factors influencing the position precision of the pushing chain: hinge clearance $e_{d i}$ and machining error $e_{l i}$. According to the mathematical model of the position precision, the influence of each factor is obtained through numerical simulations. Besides, the bended deformation is calculated using the mechanics of materials method. Based on the influences and bended deformation of the extended chain, an error compensation method is proposed to improve the position precision of chain head for a certain pushing distance. Furthermore, the pushing chain with compensation is possible to be extended in a straight line.

\section{REFERENCES}

[1] HyoJik Lee, Byung Suk Park, Ki Ho Kim. Telescopic Tube Set for Bridge Transport System. Patent 8231096B2, USA. 2011.

[2] Donghun Lee, Doyoung Chang, Yong-il Shin, Donghoon Son and Tae-wan Kim. Design and Application of a Wire-driven Bidirectional Telescopic Mechanism for Workspace Expansion with a Focus on Shipbuilding Tasks. Advanced Robotics, 25(2011), 699-715, 2011.

[3] Mark L. Enders and David J. Green. Pyrotechnic Safety Device with Retractable Telescoping Mechanism. Patent 7357415B2, USA. 2010.

[4] Kenneth J. Zimmer, Thomas J. Loomans. Single Beam Aerial Work Platform. Patent 5476050, USA. 1984.

[5] Ichiro Kawabuchi, Woo-Keun Yoon and Testsuo Kotoku. Linearmotion Telescopic Mechanism and Robot Arm Having Linearmotion Telescopic Mechanism. Patent 20120024091A1, USA, 2012 .

[6] I. Troedsson and L. Vedmar. A Dynamic Analysis of the Oscillations in a Chain Drive. ASME J. Mech. Des.,123, 395-401, 2001 .

[7] M. Chew. Inertia Effects of a Roller-Chain on Impact Intensity. ASME J. Mech. Transm. Autom. Des., 107, 123-130, 1985.

[8] Xu Lixin, Yang Yuhu, Chang Zongyu, Shen Zhaoguang and Liu Jianping. Clearance Influence on Dynamic Response of Intermittent Roller Chain Drive. Chinese Journal of Mechanical Engineering, 23(6), 699-708, 2010.

[9] Zheng. H, Y.Y. Wang and K.P. Quek. A Refined Numerical Simulation on Dynamic Behavior of Roller Chain Drives. Shock and Vibration, 11(2004), 573-584, 2004

[10] Pedersen. SL. Modeling of Contact Between Rollers and Sprockets in Chain-drive Systems. Archive of Applied Mechanics, 71(7), 489-508, 2005. 